\title{
Brain Tissue Model Classification for Telesurgery Navigation
}

\author{
Suárez B. Marco, Cifuentes G. Carlos, Suárez B. Juan, and Salinas V. Kathleen
}

\begin{abstract}
This work presents a multi-classification model of brain tissue in a simulation stage magnetic resonance imaging (MRI). Its purpose is to improve the quantification of brain pathologies and the planning of neurosurgeries. This paper shows the development and evaluation of the multi-class classification methods one-versus-one (1-v-1) and one-versus-all (1-v-r), based on support vector machines, selecting four classes of brain tissues in the sequences T1, T2, and DP (multispectral) MRI. The classified tissues were gray matter, white matter, cerebrospinal fluid (CSF) and a group of tissues called 'the rest', composed of bone, skin, muscle, fat, connective tissue and background. Finally, the performance of the classifier on different MRI slices was evaluated and showed an accuracy rate of $99.01 \%$ using the one-versus-one model, and an average of $96.65 \%$ using the one-versus-all model
\end{abstract}

Index Terms-Magnetic resonance imaging (MRI), support vector machines, brain tissue.

\section{INTRODUCTION}

Brain tissue classification is used in clinical researching and studies in the neurological field in order to monitor and quantify pathologies such as cerebrovascular accidents, cerebral edema, tumors and degenerative diseases like Parkinson's or Alzheimer's [1]. There are also applications of this method in the field of neurosurgery, both conventional and remotely operated by robots. For example, NeuroArm which is compatible with MRI [2] - and Hexapod [3] need detailed information about the patient's general anatomy, any indication of clear pathologies, geometrical information for pre-operation planning, and the generation of virtual anatomic models in order to navigate in telesurgery systems (surgery operated by remote robots).

The telesurgery system was endorsed in the United States by the Food and Drug Administration (FDA) in July 2000 [4] Technically speaking, telesurgery does not mean surgeon robots, but rather tools operated remotely by a surgeon articulated arms controlled from a console replete with a high-definition camera that produces three-dimensional images [5]. Baltimore's Johns Hopkins University Hospital has developed an integrated system for the planning, navigation and assistance of robots in skull base surgery. In the planning process, a pre-operatory tomography defines the perforation zone. This procedure results in a deviation in the planned slice of $0.6 \mathrm{~mm}$, which is clinically unacceptable, and therefore a more accurate digital analyses in pre-operatory images is required [6].

Manuscript received August 20, 2014; revised October 29, 2014. This work was supported in part by the ECCI University of Colombia.

The authors are with Espíritu Santo University of Brazil, Brazil (e-mail msuarezb@ ecci.edu.co, cacifuentes@gmail.com, suarez.juan@ecci.edu.co, salinas.kathleen@gmail.com).
At the University of Texas Southwestern Medical Center, robotic-assisted radiosurgery on brain tumors demands geometrical accuracy in the planning process, in order to minimize the risk of toxicity on healthy tissues. This can be achieved with high accuracy rates through automatic brain tissue classification [7]. The National Neuroscience Institute in Singapore proposes the use of digital image processing in the planning stage, in order to increase accuracy in the positioning and trajectory of telesurgery [8], [9].

Currently, these systems have been tested on cadavers and dogs in order to achieve precision in surgical planning [6], [8], [9]. It is precisely at this point that automatic brain tissue classification provides tools to obtain accuracy in surgical slices, to protect and prevent damages in the neurovascular system, and to improve each and every aspect that allow for scientific advancement in telesurgery.

One finds a number of different brain tissue classification techniques in the analysis and digital processing of medical images, such as K-means [10] Fuzzy C-means (FCM) [11], K-Nearest Neighbor (KNN) [12], Markov Random Fields (MRF), and Partial Volume Estimation (PVE) [13]. These are just a few of the existing classification techniques. The development of this work is usually made on a single MRI sequence, starting with the extraction of the cranial cortex in order to eliminate interference in the classification [10], [12].

The classification of brain tissue in MRI originates from: a) different image intensities, b) spatial modeling, and/or c) volumetric estimation. These classifiers entail expensive training and use multiple local minima [14].

One of the most-used techniques is the Support Vector Machine (SVM), based on the statistical learning theory [15]. SVMs were initially developed to solve binary problems, and later extended in use to solve multi-class problems [16], [17]. SVMs allow for an optimal solution for a classification problem with small sample size, leading to a global solution that not only overcomes the problem of multiple local minima, but also transfers the problem into a higher dimensional space and attains a non-linear classification in the original space. This classification method has been proven to work better than other similar classic methods [18].

The SVM classification method assumes a training data set $\left(x_{i}, y_{i}\right), i=1, \cdots: ; ; 1$ where $x_{i} \in \mathfrak{R}^{N}$ and $y_{i} \in\{-1,1\}$, which can be separated by a hyperplane $h(x)=(w \cdot x)+b=0$. In the linear case, a hyperplane is built which separates the two classes. Thus, $(\gamma)$ is the maximum-margin between the optimal hyperplane and the closest points (support vectors). This problem can be described as (1):

$$
\left\{\begin{array}{c}
\min \frac{1}{2}\|w\|^{2} \\
\text { s. } a \therefore y_{i}\left(\left(w \cdot x_{i}\right)+b\right) \geq 1
\end{array}\right.
$$


In the non-linear case (where the maximum-margin hyper plane is inserted into a transformed feature space), the entry space is inserted into a one of greater dimension to obtain new data mapping in order to use a linear classifier. In mathematical terms, non-negative variables $\xi_{i} \geq 0 \quad \forall_{i}$ and a cost parameter (C) are introduced in Equation (1). This is expressed as Eq. (2):

$$
\left\{\begin{array}{c}
\min \frac{1}{2}\|w\|^{2}+C \sum_{i} \xi_{i}^{k} \\
\text { s. } a \therefore y i\left(w \cdot x_{i}+b\right) \geq 1-\xi_{i} \\
\xi_{i}>0 \forall_{i}
\end{array}\right.
$$

Multi-class SVM classifiers are the combination of various binary classifiers. Even though there are different ways to combine these classifiers, this paper only uses the one-versus-one (1-v-1) and the one-versus-all (1-v-r) methods. In the first method, $\left(k^{*}(k-1) / 2\right)$ binary classifiers are constructed, where $k$ is the number of classes; a voting strategy for each binary classifier is used, and the class with the most number of votes is selected (Hsu \& Lin) (Smola, 2009). In the second method (1-v-r), $k$ binary models are built samples of the $i$-th class are assigned positive labels and those of other classes are assigned negative labels, where each classifier partial function fi, faces the vectors of class $\theta \mathrm{i}$ against the rest of the classes $(k-1)$.

The method developed in this research is based on SVMs and uses the multi-class techniques (1-v-1) and (1-v-r). This paper presents a model for the classification of four classes of brain tissue based on differing MRI intensities: gray matter (GM), white matter (WM), cerebrospinal fluid (CSF) and a group of tissues called 'the rest', composed of bone, skin, muscle, fat, connective tissue and background. MRI brain tissue classification is performed in three sequences: T1 (longitudinal relaxation time), T2 (transverse relaxation time) and proton density (PD). A cranial cortex extraction is not performed on these images.

This paper presents an MRI study based on the SVM classifiers. In the first part, the classifier and its method of use are explained, along with an introduction of the features of the image and the database used to evaluate the selected and designed classifier. In the second section, the results of the methods (1-v-1) and (1-v-r) are presented. The next section analyzes and discusses the obtained results, where the contributions and limitations of the model are presented. Finally, the future direction of this research is presented.

\section{MATERIALS AND METHODS}

For the realization of this research, every algorithm was programmed in Matlab® Additionally, they were supplemented using the LIBSVM library [19]. The MRI database was taken from the BRAINWEB simulator from the BIC lab [20]. In this simulated brain database, one can use three different sequences (T1, T2, PD), five slice thicknesses, six noise levels, and three levels of intensity non-uniformity. It is also possible to request personalized simulations with different parameters and to download the data from the anatomical brain model. On BRAINWEB's website different MRI images, in black and white or hot metal, can be visualized or downloaded in a MINC (.mnc) format, a volumetric format organized like so: $181,217,181$, in planes $\mathrm{X}, \mathrm{Y}, \mathrm{Z}$ (axial, coronal, and sagittal). This paper was developed in three stages: Selection of the Training Data Set, Classifier Design, and Evaluation.

\section{A. Selection of the Training Data Set}

In order to obtain the SVM training dataset, the anatomical BRAINWEB model was used. This is a fuzzy model based on Fuzzy C-Means, with manual corrections made by experts. It is also represented by volume data which defines the special distribution of different tissues such as: GM, WM, CSF, fat, muscle, skin, bone, blood vessels, connective tissue, dura mater, bone marrow and background, where the voxel intensity is proportional to the fraction of the tissue inside the voxel. The base image of this model is $\mathrm{T} 1$ with TE/TR/FA = $18 \mathrm{mS} / 10 \mathrm{mS} / 30 \mathrm{deg}$ and spatial resolution $=1 \mathrm{~mm}$, in ideal conditions ( $0 \%$ noise and $=\%$ intensity non-uniformity). In order to obtain T2 and PD data, personalized simulations with these same specifications were requested.

A pixel-by-pixel comparison is made between the tissues from the anatomic model and the reference images (T1, T2 and PD). This is made in order to determine the intensities of each tissue in the different sequences. Finally, intensities for the slices 40, 90, 120 are obtained with the purpose of having a number of small samples, thereby reducing the computational cost.

For classifier training, 4500 random samples were taken from the previously acquired tissues (GM, WM, CSF), and 900 random samples of each tissue were also taken to shape the class called 'the rest' (bone, skin, muscle, fat, meninges and background). This way, computational costs and time are reduced when using the classifier. Fig. 1 represents the space of the training data set.

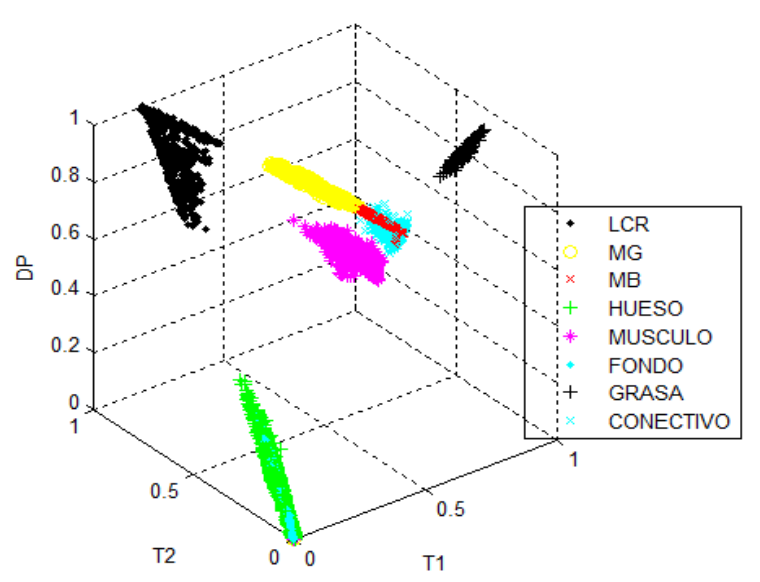

Fig. 1. Training data set sample.

\section{B. Classifier Design}

In this brain tissue classification proposal, multi-class SVM classification methods (1-v-1 and 1-v-r) are used. In its development, labels are assigned to the data samples, previously acquired for SVM training, as shown in Table I.

Parameters $\mathrm{C}$ and $\gamma$ are designed using cross-validation (CV) with the grid search, which creates a 'web' of every possible location of the intersection points of configuration in order to find the best parameters. The $\mathrm{C}$ values $\mathrm{C}=2-5$, $2-3, \ldots, 215$ and $\gamma=2-15,2-13, \ldots, 23$ were assigned, evaluating different kernel functions: linear, polynomial, radial basis function (RBF) and sigmoid. RBF was found to 
have the best performance. The one-versus-one method was trained with the best results found from the CV: $\mathrm{C}=32768, \gamma$ $=8$, with a classification accuracy of $98.8222 \%$. In the classification method one-versus-all, four binary classifiers were established, contrasting one tissue with the remaining ones. The layout of the four classifiers is shown in Table II. The grid search was applied independently to each one of these classifiers in order to find the optimal performance parameters. The values with the most accurate classification are presented in Table III. Finally, predictions about the test images are made with both methods.

\begin{tabular}{lll} 
TABLE I: TRAINING DATA SET LABELS \\
\hline \hline Tissue & Class & Label \\
\hline WM & WM & 1 \\
GM & GM & 2 \\
CSF & CSF & 3 \\
Bone & Rest & 4 \\
Skin and Muscle & Rest & 4 \\
Background & Rest & 4 \\
Connective & Rest & 4 \\
Fat & Rest & 4 \\
\hline \hline
\end{tabular}

\begin{tabular}{cll}
\multicolumn{3}{c}{ TABLE II: BINARY CLASSIFIERS IN 1-V-R } \\
\hline \hline Model & Class 1 & Class 2 \\
\hline 1 & GM & WM, CSF and rest \\
2 & WM & GM, CSF and rest \\
3 & CSF & GM, WM and rest \\
4 & Rest & GM, WM and CSF \\
\hline \hline
\end{tabular}

TABLE III: CV BINARY CLASSIFIERS

\begin{tabular}{cccc}
\multicolumn{4}{c}{ TABLE III: CV BINARY CLASSIFIERS } \\
\hline \hline Model & $\boldsymbol{\Gamma}$ & C & \% Classification \\
\hline 1 & 8 & 0.03125 & 99.5882 \\
2 & 8 & 32 & 98.8111 \\
3 & 8 & 0.0125 & 100 \\
4 & 8 & 32768 & 98.3111 \\
\hline \hline
\end{tabular}

\section{Classifiers Evaluation}

In order to evaluate the multiclass classification methods, MRI was introduced into the sequences T1, T2 and PD. The initial test was made with axial section 90, a medium training cut, in which there is more GM and WM presence, to establish its behavior in ideal conditions.

Later, tests were made in axial sections different from the training sections, as follows. Section 30 was chosen for its greater presence of bone, muscle and skin; section 105 was chosen because the cerebellum starts under section 75 and in the higher sections there is higher presence of CSF. These slices are evaluated using a confusion matrix (CM) which establishes the false positives and the false negatives of a classification. In this analysis the labels of the training data sets and the predicted data are evaluated. The data is represented in $\mathrm{N} \times \mathrm{N}$ matrices that correspond with the classes of the classification, establishing the accuracy rate for each tissue.

\section{RESULTS}

Taking into account that the classifier has been trained in ideal conditions, tests with low levels of noise and intensity non-uniformity are performed. These conditions would be equivalent to the best conditions found in the creation of real images. The test images have the following parameters: thickness $=1 \mathrm{~mm}, 1 \%$ noise and $20 \%$ intensity non-uniformity. The classifiers' response is presented in binary images and the quantitative evaluation is made using the CM. Fig. 2 shows the different MRI sequences that were inserted into the classifier.

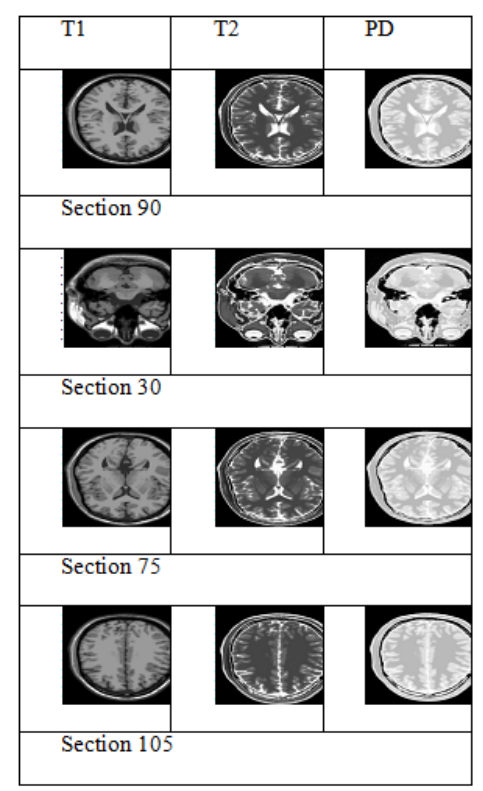

Fig. 2. Input images in T1, T2 and DP sequences, in the axial sections 90, 30, 75 and 105.

Images in Fig. 3 correspond to the response in the one-versus-one method. Fig. 4 shows the results of the classification made using the one-versus-all method. In these binary images each tissue class is shown independently in each different test section.

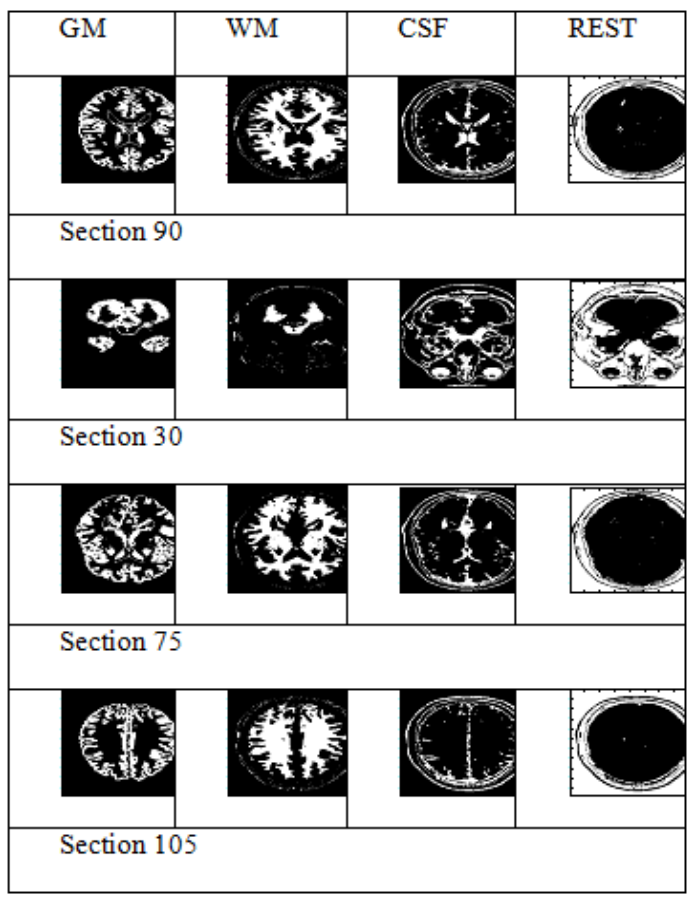

Fig. 3. Multiclass SVM one-versus-one results in sections 90, 30. 75 and 105.

The evaluation is done using the $\mathrm{CM}$ in each test section. The data is represented in $4 \times 4$ matrices that correspond to the 
classification classes, establishing the accuracy rate for each tissue. The CM average of the test sections are shown in Table IV for both classifiers.

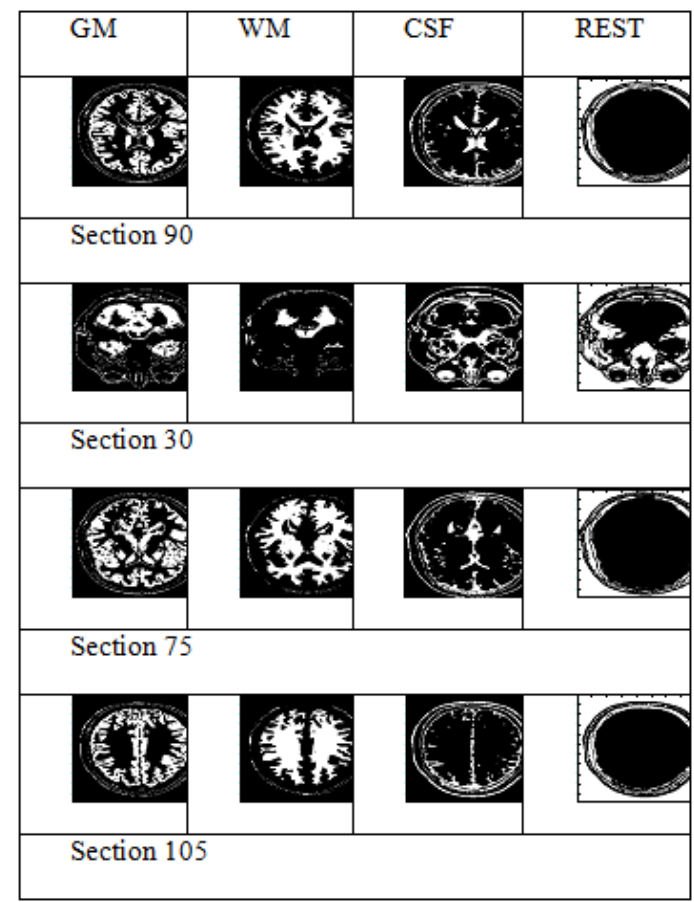

Fig. 4. Multiclass SVM one-versus-all results in sections 90, 30. 75 and 105.

TABLE IV: CLASSIFIER CONFUSION MATRIX

\begin{tabular}{ccccc}
\hline \hline \multicolumn{5}{c}{ 1-v-1 } \\
\hline \hline & GM & WM & CSF & Rest \\
GM & 0.999775 & 0.000225 & 0 & 0 \\
WM & 0.000075 & $0, .96775$ & 0 & 0.00315 \\
CSF & 0 & 0 & 1 & 0 \\
Rest & 0.0499 & 0.0388 & 0.1485 & 0.7628 \\
\hline \hline \multicolumn{5}{c}{ 1-v-r } \\
\hline \hline & GM & WM & CSF & Rest \\
GM & 0.999025 & 0.000925 & 0.00005 & 0 \\
WM & 0.000075 & 0.9979 & 0 & 0.002025 \\
CSF & 0 & 0 & 1 & 0 \\
Rest & 0.077825 & 0.04225 & 0.146575 & 0.73335 \\
\hline \hline
\end{tabular}

The time that it takes to make the prediction is calculated after the training of each model. The algorithm is executed in a $2.20 \mathrm{GHz}$ DualCore processer with $2 \mathrm{~GB}$ of RAM. The average time for the test sections are 6.9359 seconds in the $1-\mathrm{v}-1$ method and 27.1383 seconds in the $1-\mathrm{v}-\mathrm{r}$ classifier.

\section{DISCUSSIONS}

The performed classification of brain tissue shows high accuracy rates and reduced execution times. The result obtained in the GM class in each section with the 1-v-1 method appears totally independent from the extra cranial tissue, even in the lowest section (30). In this section the independence is also clear, despite having more presence of bone and muscle, which makes the classification more difficult. Meanwhile, in the method 1-v-r the tissue shows addition of extra cranial tissue.

In the classification of the tissues WM and CSF, similar performance is observed of both classifiers. However, the addition of extra cranial tissues is clear due to similarities in intensity in both models. In future research, the intracranial tissues adjacent to the bone (bone marrow and connective tissue) must be taken into account in order to improve the classifier's performance.

The quantitative analysis of the performed classification via the CM confirms the precision in the classification of the tissues GM, WM and CSF. Likewise, it allows error-checking in the detection of the 'the rest' class in other classes of tissues. This analysis shows, as well, the superiority of the 1-v-1 method over the 1-v-r one in every class of established tissue. The 1-v-r classifier can be improved by establishing a different voting scheme.

The generation of new labeling from a fuzzy model with manual correction allows one to work with any MRI sequence, with high accuracy rates. Even though there are classifiers with higher accuracy rates (like the one used in 3D reconstruction, with the PVE technique, implemented in a FPGA), this one only accepts the T1 sequence [12].

3D reconstructions with Immune Sphere-Shaped SVMs, where 7 classes of tissues are evaluated [17], use an immune algorithm in order to find the best parameters for $\mathrm{C}$ and $\gamma$. In the case of this paper, the grid search was used, which gives better accuracy rates that are verifiable in the results of the evaluation by the $\mathrm{CM}$ for the same class of tissue. Additionally, as said before in this paper, there is the possibility of classifying brain tissue from any of the three MRI sequences.

\section{FUTURE DIRECTIONS}

The research group plans to apply the results obtained from the classified images on a study of real images. This will then be applied in the quantification of pathologies and the planning of neurosurgery. It will also be of great help in research about accuracy in telesurgery positioning and trajectory through the 3D model generation of anatomic structures for virtual navigation. Regarding the study of real images, the research group proposes the incorporation of an image base from patients at the University Clinic of La Sábana from Colombia.

\section{REFERENCES}

[1] A. Ramani, J. H. Jensen, and J. A. Helpern, "Quantitative MR imaging in Alzheimer disease," Radiology, vol. 241, no. 1, pp. 26-44, 2006.

[2] New Scientist Tech and Reuters. NewScientist. (2009, April) MRI-enabled brain surgery robot revealed. [Online]. Available: http://www.newscientist.com/article/dn11631-mrienabled-brain-surge ry-robot-revealed.html

[3] DPA. (2003). World's first robot brain surgeon developed. [Online]. Available: http://www.smh.com.au/articles/2003/01/15/1042520673704.html

[4] W. S. Melvin, J. M. Dundon, M. Talamini, and S. Horgan, "Computer-enhanced robotic telesurgery minimizes esophageal perforation during Heller myotomy," Surgery, vol. 138, no. 4, pp. 553-559, 2005.

[5] S. L. Lee, M. Lerotic, V. Vitiello, S. Giannarou, K. Kwok, M. Visentini-Scarzanella, and G. Z. Yang, "From medical images to minimally invasive intervention: Computer assistance for robotic surgery," Computerized Medical Imaging and Graphics, vol. 34, no. 1, pp. 33-45, 2010.

[6] G. Tian, Y. Xia, Y. Zhang, and D. Feng, "Hybrid genetic and variational expectation-maximization algorithm for gaussian-mixture-model-based brain MR image segmentation," IEEE 
Transactions on Information Technology in Biomedicine, vol. 15, 373-380, 2011.

[7] C. A. Giller, F. Raschke, N. P. Davies, M. Wilson, A. C. Peet, and F. A. Howe, "Classification of single - voxel $1 \mathrm{H}$ spectra of childhood cerebellar tumors using lcmodel and whole tissue representations," Magnetic Resonance in Medicine, vol. 70, no. 1, pp. 1-6, 2013.

[8] S. K. Chang, W. W. Hlaing, L. Yang, and C. K. Chui, "Current technology in navigation and robotics for liver tumours ablation," Annals of the Academy of Medicine-Singapore, vol. 40, no. 5, pp. 231-236, 2011.

[9] J. P. Kobler, J. Kotlarski, J. Öltjen, S. Baron, and T. Ortmaier, "Design and analysis of a head-mounted parallel kinematic device for skul surgery," International Journal of Computer Assisted Radiology and Surgery, vol. 7, no. 1, pp. 137-149, 2012.

[10] Y. Fan, D. Shen, R. C. Gur, R. E. Gur, and C. Davatzikos, "COMPARE: classification of morphological patterns using adaptive regional elements," IEEE Transactions on Medical Imaging, vol. 26, no. 1, pp. 93-105, 2007.

[11] P. L. Bazin and D. L. Pham, "Topology-preserving tissue classification of magnetic resonance brain images," IEEE Transactions on Medical Imaging, vol. 26, no. 4, pp. 487-496, 2007.

[12] P. Anbeek, K. L. Vincken, G. S. Van Bochove, M. J. Van Osch, and J. van der Grond, "Probabilistic segmentation of brain tissue in MR imaging," Neuroimage, vol. 27, no. 4, pp. 795-804, 2005.

[13] J. J. Koo, A. C. Evans, and W. J. Gross, "3-D brain MRI tissue classification on FPGAs," IEEE Transactions on Image Processing, vol. 18, no. 12, pp. 2735-2746, 2009

[14] Y. Fan, D. Shen, and C. Davatzikos, "Classification of structural images via high-dimensional image warping, robust feature extraction, and SVM," Medical Image Computing and Computer-Assisted Intervention-MICCAI, Springer Berlin Heidelberg, 2005, pp. 1-8.

[15] C. Cortes and V. Vapnik, "Support-vector networks," Machine Learning, vol. 20, no. 3, pp. 273-297, 1995.

[16] C. W. Hsu and C. J. Lin, "A comparison of methods for multiclass support vector machines," IEEE Transactions on Neural Networks, vol. 13 , no. 2 , pp. 415-425, 2002.
[17] A. J. Smola, C. Schölkopf, A. Burges, and T. Joachims, "Making large-Scale SVM learning practical," Advances in Kernel Methods Support Vector Learning. s.1. : MIT-Press, 2009.

[18] L. Guo, X. Liu, Y. Wu, W. Yan, and X. Shen, "Research on the segmentation of MRI image based on multi-classification support vector machine," in Proc. 29th Annual International Conference of the IEEE Engineering in Medicine and Biology Society, pp. 6019-6022, 2007.

[19] C. C. Chang and C. J. Lin, "LIBSVM: A library for support vector machines," ACM Transactions on Intelligent Systems and Technology (TIST), vol. 2, no. 3, 2011.

[20] Brain Imagin Center-Monterreal Neurology Institute-McGill. (2006) BrainWeb: Simulated Brain Database. [Online]. Available: http://mouldy.bic.mni.mcgill.ca/brainweb/.

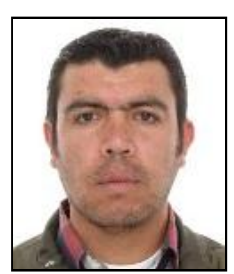

Suárez B. Marco is researcher at ECCI University from Bogota Colombia. He was born in Duitama Colombia. His works focus on subjects about machine learning, semantic web and knowledge Discovery. $\mathrm{He}$ is currently doing Ph.D studies at UPAEP Mexico.

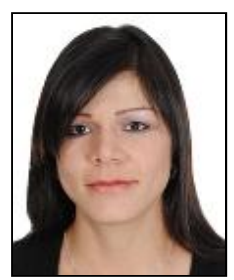

Salinas V. Kathleen is an assistant professor at High Office Educational of the Boyacá Department. She was born in Paipa Colombia. Her research areas are management technology and knowledge management. She has received master's degree in information technology management at UNINI Puerto Rico. 